\title{
Influence of optical path delay control on a ground-based nulling interferometer
}

\author{
Olivier Absil $^{a, b}$ and Vincent Coudé du Foresto ${ }^{b}$ \\ ${ }^{a}$ IAGL, University of Liège, B5C, 17 Allée du 6 Août, B-4000 Sart-Tilman, Belgium \\ ${ }^{b}$ LESIA, Observatoire de Paris-Meudon, 5 place J. Janssen, F-92195 Meudon, France
}

\begin{abstract}
Atmospheric turbulence is a major source of noise in any fiber-linked interferometer through the piston effect between the two arms of the interferometer which induces an erratic movement of the fringes. Because the stellar light has to be permanently cancelled by the central dark fringe, a ground-based nulling interferometer is not possible without stringent optical path delay (OPD) control. In this paper, we investigate the influence of the residual OPD error of a fringe tracking unit on the performances of a nulling interferometer. The accuracy required for this control system strongly depends on the observation wavelength. If we want to detect exozodiacal clouds ten times as dense as our zodiacal dust cloud in the near-infrared ( $\mathrm{L}$ ' band), the performances of the fringe tracker are crucial: the residual OPD should be about 20 nanometers RMS. This specification can only be reached if the fringe sensor is optimized for bright sources. In the mid-infrared ( $\mathrm{N}$ band), the requirements are strongly relaxed: OPD control with an accuracy of $400 \mathrm{~nm}$ RMS is sufficient to be background-limited, but a lower residual OPD (about $50 \mathrm{~nm}$ RMS) is strongly recommended to reduce the stellar leakage.
\end{abstract}

Keywords: Nulling interferometry, infrared, atmospheric turbulence, OPD control, intensity control

\section{INTRODUCTION}

Ground-based nulling interferometers are currently being built by European and American teams: the GENIE experiment will use the infrastructure of the VLTI- four 8-m telescopes and three 1.8-m telescopes-in Paranal (Chile), while the Keck and LBT nullers will both recombine two telescopes in a nulling mode. The principle of nulling interferometry is two recombine the beams coming from two (or more) telescopes in order to produce a destructive interference on the axis of the interferometer. This can be done be introducing an achromatic phase shift of $\pi$ radians in one arm of the interferometer. The light from a star can thus be cancelled, revealing its close environment. The main targets for these interferometers are accretion disks around young stellar objects, exozodiacal dust clouds around nearby main-sequence stars and possibly hot Jupiters. A survey of nearby exozodiacal clouds is a major requirement for future space missions dedicated to Earth-like planet detection, such as Darwin and TPF, because their performance depends on the amount of dust around their target stars. In order to produce significant information for these space missions, the ground-based nullers should be sensitive to dust clouds about ten times as dense as our zodiacal cloud. Such a dust cloud, surrounding a Sun-like star located at 10 parsecs, will be our reference target during this study.

The three nulling interferometers mentioned above are expected to operate in the mid-infrared: the $\mathrm{N}$ band (from 8 to $13 \mu \mathrm{m}$ ), or a part of the $\mathrm{N}$ band, is the baseline for all of them. Near-infrared observations in the L' band (from 3.5 to $4.1 \mu \mathrm{m}$ ) are also considered for the GENIE instrument. The difficulties encountered in these two spectral domains are very different: the thermal background produced by the atmosphere and the instrument is the dominant source of noise in the mid-infrared, while the control of the nulling process in presence of atmospheric turbulence is the key for near-infrared observations.

In this paper, we investigate the effect of atmospheric turbulence on the nulling process, and try to deduce the performances of the control loop needed to reduce atmospheric piston down to an acceptable level. All the following developments are made for a simple two-telescopes configuration (the Bracewell ${ }^{1}$ configuration), but they could easily be generalized to a more complex interferometric array. A separation of 46 meters is assumed between the two telescopes (LBT: $14 \mathrm{~m}$, GENIE: $46 \mathrm{~m}$, Keck: $85 \mathrm{~m}$ ).

Further author information: (Send correspondence to O.A.)

O.A.: E-mail: absil@astro.ulg.ac.be, Telephone: +32 (0)4366 9761

V.C.d.F.: E-mail: Vincent.Foresto@obspm.fr, Telephone: +33 (0)1 45077963 


\section{STELLAR LEAKAGE IN NULLING INTERFEROMETRY}

The destructive interference produced by a nulling interferometer is only perfect on the axis of the interferometer. Due to the finite extent of the stellar disk, a part of the starlight leaks through the null, producing a spurious signal from which the exozodiacal signal has to be extracted. Appropriate calibration methods have to be found to handle this problem. They will be briefly discussed in section 4. Assuming that the mean stellar leakage can be removed with a good precision, our major concern in this paper will be the noise associated to the stellar leakage. This noise can be divided into two parts: the photon noise associated to the mean stellar leakage, and the "transmission noise" due to the fluctuations of the stellar transmission. These fluctuations are produced by three main sources: differential optical path delay between the arms of the interferometer, intensity mismatches due to unequal coupling efficiencies into the fibers for the two telescopes, and phase fluctuations due to water vapor dispersion. Only the first source will be discussed here.

Stellar leakage can be considered as a doubly stochastic Poisson process, which means that the photon arrival rate itself is a stochastic process. Goodman ${ }^{2}$ fully discusses the statistics of doubly stochastic Poisson processes in chapters 3 and 9. Letting $\bar{K}$ the mean number of photo-events detected during the integration time $t_{\text {int }}$ and $W$ the integrated intensity on $t_{\text {int }}$, the mean and variance of $K$ are found to be (Goodman, ${ }^{2}$ page 470 ):

$$
\bar{K}=\alpha \bar{W}, \quad \sigma_{K}^{2}=\alpha \bar{W}+\alpha^{2} \sigma_{W}^{2},
$$

where $\alpha=\eta / h \nu$ is a constant taking the quantum efficiency $\eta$ of the instrument into account. The variance $\sigma_{K}^{2}$ of $K$ thus consists of two distinct terms each of which has a physical interpretation. The first, which is proportional to the total energy incident during the measurement, can be interpreted as representing the pure Poisson noise introduced by the random interaction of light and matter. The second, because it is proportional to $\sigma_{W}^{2}$ (variance of the incident intensity between successive integration times), is the classically expected result in the absence of any noise associated with the interaction of light and matter.

In our case, the intensity $W$ is the product of the stellar flux $F_{\text {star }}$ with the stellar transmission $\tau$, which is the stochastic part of the signal. The transmission noise $\sigma_{\text {trans }}$ can thus be written

$$
\sigma_{\text {trans }}=\alpha F_{\text {star }} \sigma_{\tau}
$$

The temporal evolution of the transmission noise depends on the statistics of OPD fluctuations, which are discussed in the next section.

In order to assess the prevalence of stellar leakage fluctuations on the overall instrument performances, its contribution to the total noise must be compared with other noises, which include the photon noise from the thermal background, the exozodiacal cloud and the mean stellar leakage itself, as well as the detector noise. Background fluctuations are not considered in this paper, assuming that an efficient chopping method is available to cancel them. Estimated infrared background levels are presented in table 1, including the emission of the atmosphere and of the warm optics (a mean temperature of $288 \mathrm{~K}$ is assumed for the instrument).

\section{INFLUENCE OF OPD CONTROL}

\subsection{Atmospheric piston}

A major source of stellar leakage fluctuations is the differential optical path delay (OPD hereafter) between the two arms of the interferometer, generated by atmospheric turbulence: the non-homogeneity of the atmospheric refraction index induces a differential OPD between the two arms of the interferometer, referred to as piston. The piston is highly variable with time, with a zero mean and a typical standard deviation of $20 \mu \mathrm{m}$ RMS for a $46 \mathrm{~m}$ baseline. It is characterized by its power spectral density (PSD), which is proportional to $f^{-8 / 3}$ for frequencies higher than $f_{c} \simeq 0.2 v / B$, where $v$ is the wind speed and $B$ the baseline length, and proportional to $f^{-2 / 3}$ for $f<f_{c}$ (Ménardi and Gennai ${ }^{4}$ ). A even steeper slope $\left(f^{-17 / 3}\right)$ is expected for frequencies above $f_{c_{2}} \simeq 0.3 v / D$, with $D$ is the telescope diameter (Conan et al. $^{5}$ ), but is not included is these simulations.

The effect of differential OPD is to make the fringes move around their central position. In the case of a nulling interferometer, this means that the central dark fringe is not centered on the stellar disk any more, so that the starlight transmission increases. In the $\mathrm{N}$ band, an OPD variation of $5 \mu \mathrm{m}$ is sufficient to get a constructive 
Table 1. Estimated background flux and associated signal-to-noise ratios (SNR) for exozodiacal cloud detection in the near- and mid-infrared with an 8-m class telescope (mean sky brightness adapted from Cuby et al. ${ }^{3}$ ). The target consists of a 10-zodi cloud surrounding a Sun-like star at $10 \mathrm{pc}$. Signal-to-noise ratios only take into account the photon noise from the mean background and from the exozodiacal emission. An overall instrumental transmission of $1 \%$ is assumed to compute the estimated SNR.

\begin{tabular}{|l|c|c|c|c|}
\hline Spectral band & $\mathrm{K}$ & $\mathrm{L}$ & $\mathrm{M}$ & $\mathrm{N}$ \\
\hline Exozodiacal flux $(\mathrm{Jy})$ & $0.2 \times 10^{-3}$ & $0.5 \times 10^{-3}$ & $0.7 \times 10^{-3}$ & $1.1 \times 10^{-3}$ \\
Mean sky brightness $\left(\mathrm{Jy} / \mathrm{as}^{2}\right)$ & 0.004 & 3 & 30 & 400 \\
Blackbody at 288 K $\left(\mathrm{Jy} / \mathrm{as}^{2}\right)$ & 0.012 & 32 & 251 & 6567 \\
\hline Total background $(\mathrm{Jy})$ & $6.4 \times 10^{-5}$ & 0.42 & 5.3 & 613 \\
Estimated SNR in 100 seconds & 140 & 8 & 3 & 1 \\
\hline
\end{tabular}

interference instead of a destructive one. Therefore the differential OPD must be reduced well below $5 \mu \mathrm{m}$ by means of a Fringe Sensing Unit (FSU). The nulling process needs a very high stability in the position of the fringes. Note that pointing and tip-tilt errors also induce OPD, which will also be (partially) corrected by the FSU. In this study, we will focus on the OPD error due to atmospheric piston because it is supposed to be the dominant source of differential OPD.

\subsection{The Fringe Sensing Unit (FSU)}

In order to get an approximate model of the residual OPD power spectrum, a simple model is used for the FSU, adapted from the PRIMA Fringe Sensor Unit technical specifications ${ }^{4}$ (see Fig. 1). This model is not optimal for fringe tracking: it is only used to get an approximate PSD of the residual OPD.

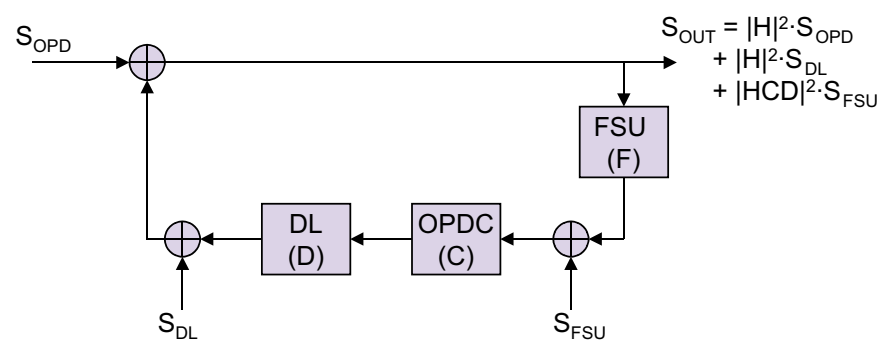

Figure 1. Block-diagram of a simple fringe tracking system. The OPD power spectrum of the atmosphere, the fringe sensing unit and the delay line are respectively written $S_{\mathrm{OPD}}, S_{\mathrm{FSU}}$ and $S_{\mathrm{DL}}$, while the total output power spectrum is $S_{\text {OUt. }}$ The transfer functions of the FSU, OPD corrector and delay line are respectively noted $F, C$ and $D$, and the overall transfer function $H$.

Assuming the OPD corrector to be a simple integrator $(C=K / s$, where $s$ denotes the Laplace variable and $K$ the integrator gain factor), the delay line to be an ideal transducer $(D=1)$ and the fringe sensing unit to act as a pure $T_{i} / 2$ time delay (where $T_{i}$ is the FSU integration time), the closed loop transfer function writes:

$$
H(s)=\frac{1}{1+F C D}=\frac{s}{s+K \exp \left(-s T_{i} / 2\right)} .
$$

With this transfer function, the low frequency part of the atmospheric OPD is well reduced by the feedback loop, while the high frequency components are unaffected. The frequency at which the transfer function reaches unity is $f_{K}=K / 2 \pi$. The larger the gain, the better the OPD correction. However, the gain should not be larger than $0.5 / T_{i}$ in order to keep the loop stable. Fig. 2 shows the initial and corrected power spectral densities of OPD 
sources. The OPD induced by the delay lines has not been taken into account in these simulations because data were not available at this time, but it is not expected to be dominant. With a repetition frequency of $1 \mathrm{kHz}$, the residual RMS OPD, which is the area under the dashed line, is equal to about $60 \mathrm{~nm}$. This is approximately the residual OPD currently expected for the PRIMA fringe sensor at the VLTI.

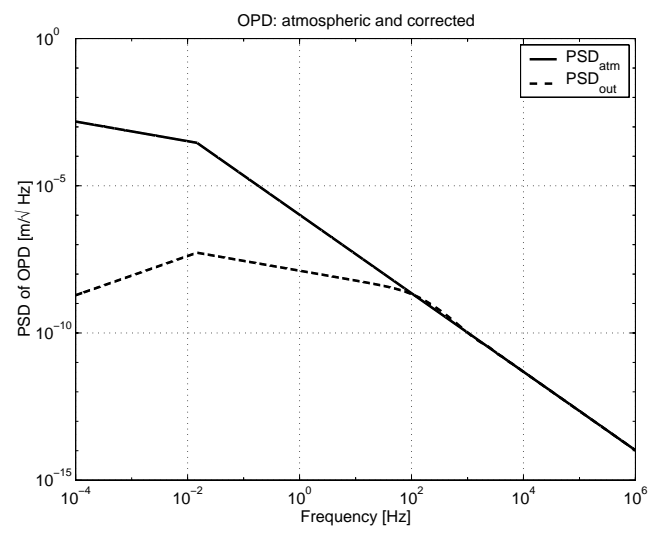

Figure 2. Power spectral density of the atmospheric OPD and of the residual OPD at the output of the Fringe Sensing Unit. Delay line noise has not been included in this simulation.

\subsection{Associated stellar transmission}

As expected, the differential OPD makes the central fringe move around its central position, which is the center of the stellar disk since we assume the telescope pointing to be perfect. The fringe motion is illustrated in Fig. 3 . Its effect is two-fold: it decreases the final cloud/star ratio by increasing the mean stellar transmission, and it introduces noise associated with the stellar leakage fluctuations.
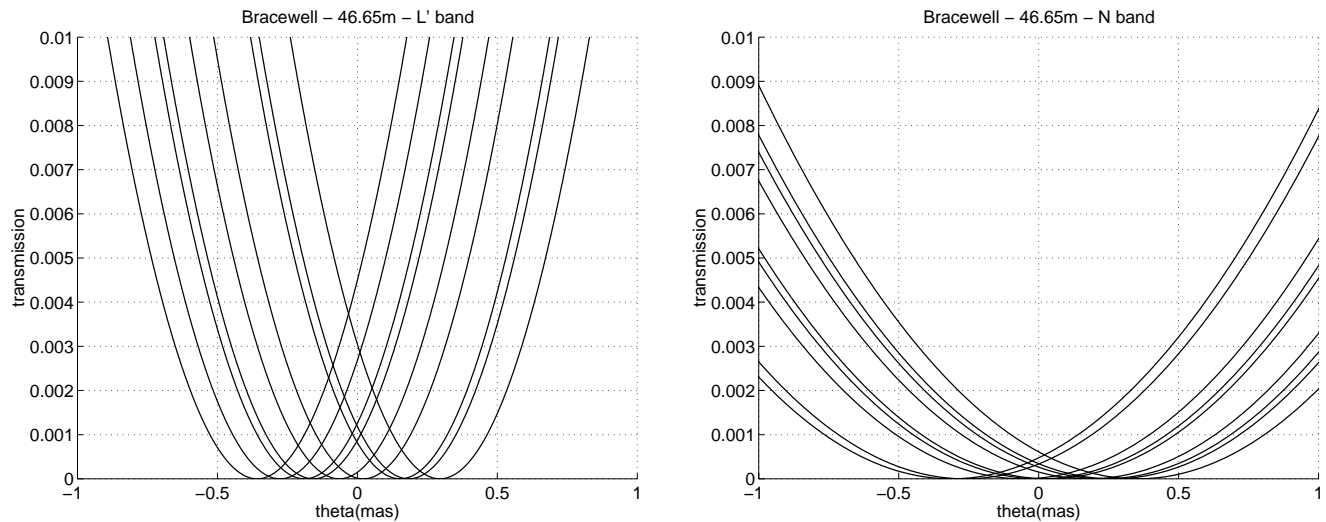

Figure 3. Movement of the central dark fringe for a residual OPD of $60 \mathrm{~nm}$ RMS, in the L' band (left) and in the N band (right). The displacement of the central fringe on the sky plane is related to the differential OPD by $\theta=\Delta \mathrm{OPD} / B$, so that the RMS displacement is of 0.26 mas. In comparison, the angular radius of a Sun at 10 pc is about 0.5 mas.

The stellar transmission associated to the residual OPD of the FSU is plotted in Fig. 4 for two wavelengths ( $\mathrm{L}$ ' and $\mathrm{N}$ bands). The transmission curves have a very characteristic shape: large fluctuations are added on top of a constant transmission which would be observed in the absence of OPD fluctuations. This theoretical stellar transmission depends on the wavelength because the fringe width scales as the wavelength. It is equal to $1.3 \times 10^{-3}$ in the $\mathrm{L}^{\prime}$ band and to $1.7 \times 10^{-4}$ in the $\mathrm{N}$ band. The nulling process is much more efficient in the $\mathrm{N}$ band where the central fringe is about three times wider. This adds to the fact that the initial contrast between 
the star and the exozodiacal cloud is lower in the $\mathrm{N}$ band than in the $\mathrm{L}$ ' band (see table 3 ), making the $\mathrm{N}$ band much less sensitive to stellar leakage than the L' band. However, this does not necessarily mean that the $\mathrm{N}$ band is better because other sources of noise have to be considered.
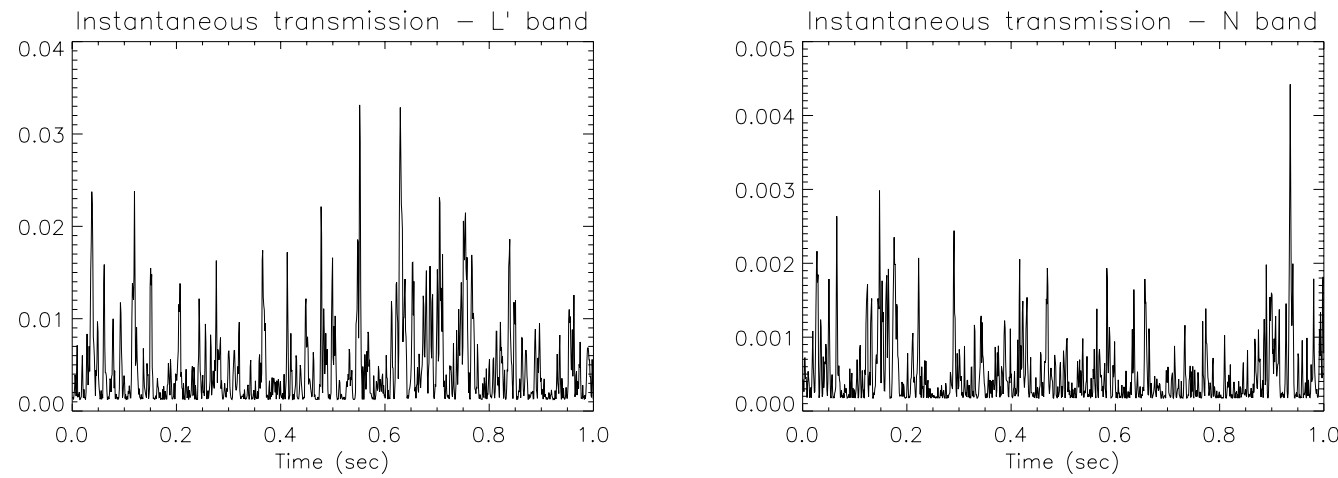

Figure 4. Instantaneous stellar transmission due to a residual OPD of $60 \mathrm{~nm}$ RMS, in the L' band (left) and in the N band (right), for a Bracewell interferometer $46 \mathrm{~m}$ long observing a Sun-like star at $10 \mathrm{pc}$. The N-band transmission is about eight times lower than the L'-band transmission.

In Fig. 5 are plotted the three types of noise considered here: the transmission noise due to stellar leakage fluctuations (dashed line), the photon noise from the background, mean stellar leakage and exozodiacal cloud (solid line), and the detector noise (dashed-dotted line). These two plots are very instructive: they show that with the expected fringe tracking performances of PRIMA, L'-band observations are limited in signal-to-noise ratio by the transmission noise, while N-band observations are dominated by the photon noise from the background-and also possibly by background fluctuations which are not included here. The overall signal-to-noise ratio in 100 seconds of integration is of 0.6 in the L' band and of 0.4 in the $\mathrm{N}$ band. Even if the SNR are about the same, a big difference is that L'-band observations are very sensitive to the actual performance of the FSU, as shown in the next paragraph.
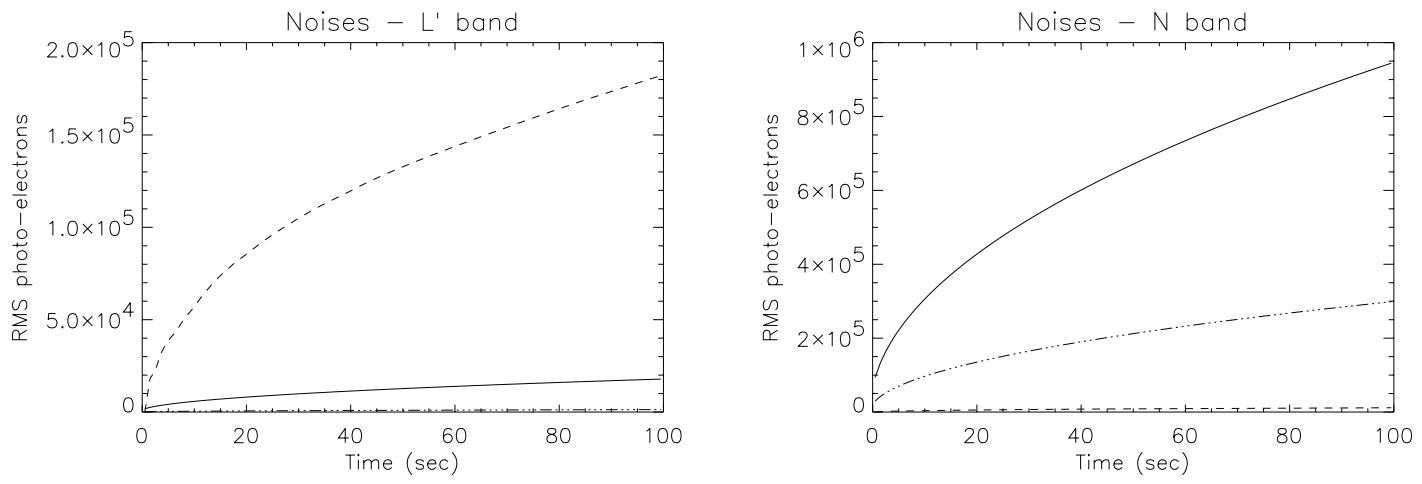

Figure 5. Noise contribution from the stellar leakage fluctuations (dashed line) with respect to integration time, compared to the photon noise from the mean background, mean leakage and exozodiacal cloud (solid line) and to the detector noise (dash-dotted line). Simulations carried out in the L' band (left) and in the N band (right).

\subsection{Influence of the FSU performances}

The simulated performances of next-generation Fringe Sensing Units are still speculative. Moreover, these FSU's are not expected to be optimized for the bright targets considered in nulling interferometry, where the central 
star generally has a visual magnitude of about 3. One could therefore think of implementing a second, more accurate stage of OPD control inside the nulling instrument. Preliminary simulations show that a residual OPD of about $10 \mathrm{~nm}$ RMS is reachable. Table 2 demonstrates that the signal-to-noise ratio in the L' band is very sensitive to the FSU performances, and that it could be as high as 7.5 in 100 seconds if the fringe sensor was optimized for bright targets.

Table 2. Influence of the FSU repetition frequency $\left(f_{r}\right)$ on the performances of a Bracewell interferometer. The target consists of a 10-zodi disk surrounding a Sun-like star at $10 \mathrm{pc}$ (as usual). The signal-to-noise ratios include the three types of noise (transmission noise, photon noise and detector noise) and are computed for an integration time of 100 seconds.

\begin{tabular}{|c|c|c|c|c|c|}
\hline & & \multicolumn{2}{|c|}{ Mean stellar transmission } & \multicolumn{2}{c|}{ SNR in $100 \mathrm{sec}$} \\
\hline$f_{r}$ & $\sigma_{\text {OPD }}$ & L' band $^{\prime}$ & N band & L' band & N band \\
\hline $0.5 \mathrm{kHz}$ & $100 \mathrm{~nm}$ & $7.8 \times 10^{-3}$ & $1.3 \times 10^{-3}$ & 0.2 & 0.4 \\
$1.3 \mathrm{kHz}$ & $50 \mathrm{~nm}$ & $3.0 \times 10^{-3}$ & $4.6 \times 10^{-4}$ & 1 & 0.4 \\
$3 \mathrm{kHz}$ & $25 \mathrm{~nm}$ & $1.6 \times 10^{-3}$ & $2.4 \times 10^{-4}$ & 5 & 0.4 \\
$10 \mathrm{kHz}$ & $10 \mathrm{~nm}$ & $1.3 \times 10^{-3}$ & $1.9 \times 10^{-4}$ & 7.5 & 0.4 \\
\hline
\end{tabular}

Fig. 6 shows that for a residual OPD of $25 \mathrm{~nm}$ RMS, the transmission noise becomes comparable to the background noise in the L' band. For lower residual OPD's (i.e. for better FSU performances), the background becomes dominant and the signal-to-noise ratio does not increase much more. This indicates that a residual OPD of about $20 \mathrm{~nm}$ RMS is sufficient to ensure background-limited observations in the L' band. On the other hand, $\mathrm{N}$ band observations are always background-limited: the transmission noise becomes dominant only if the residual OPD exceeds $400 \mathrm{~nm}$ RMS. This is due to the huge thermal background encountered at $10 \mu \mathrm{m}$.

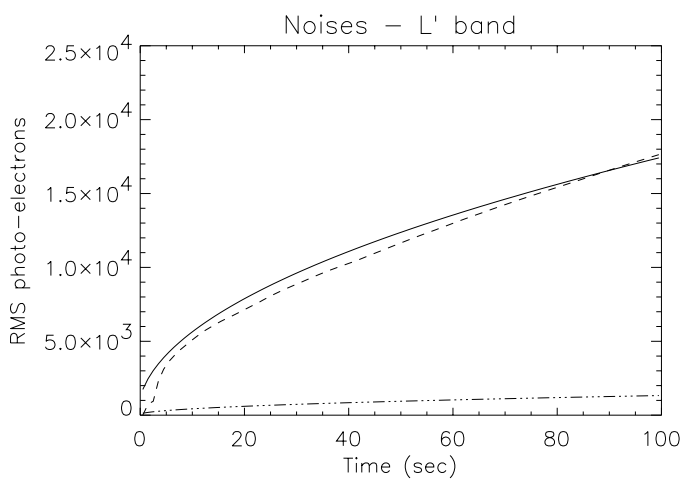

Figure 6. Noise contribution from the stellar leakage fluctuations (dashed line) with respect to integration time, compared to the photon noise from the mean background, mean leakage and exozodiacal cloud (solid line) and to the detector noise (dash-dotted line). Simulation carried out in the L' band, with a repetition frequency of $3 \mathrm{kHz}$ for the FSU, leading to a residual OPD of $25 \mathrm{~nm}$ RMS. Transmission noise and background noise become comparable in this case.

Table 2 also displays the mean stellar transmission computed for the different FSU repetition frequencies. As expected, the larger the residual OPD, the larger the stellar transmission. For a residual OPD of $10 \mathrm{~nm}$ RMS, the optimum transmission is almost reached, which means that leakage fluctuations become small compared to the mean value of the leakage. Such a behaviour would make the calibration of stellar leakage much easier.

\section{STELLAR LEAKAGE CALIBRATION}

Nulling interferometry uses pupil-plane recombination. No image is formed: we only measure the integrated flux transmitted by the interference pattern projected on the plane of the sky. Therefore, there is a priori no 
distinction between the signal from the exozodiacal cloud and other sources in the field-of-view, such as the central star. During this study, we have supposed that the mean stellar leakage signal is removed from the output signal by means of an appropriate calibration method. Let us investigate how this calibration could be done in practice. Table 3 gives the ratio of stellar to exozodiacal flux at the output of the nuller, for a realistic residual OPD (60 $\mathrm{nm}$ RMS). In the $\mathrm{N}$ band, the contrast has been reduced from 2800 to 2.5 by the nuller, while in the L' band, the contrast is still of 260 at the output of the instrument. Stellar calibration will thus be a major issue in the near-infrared, because the exozodiacal flux represents only a small part of the output signal.

Table 3. Contrast between the star and the exozodiacal cloud at the input and the output of a Bracewell nulling interferometer $46 \mathrm{~m}$ long, looking at a 10-zodi cloud surrounding a Sun-like star at $10 \mathrm{pc}$. A residual OPD of $60 \mathrm{~nm}$ RMS is assumed (repetition frequency of $1 \mathrm{kHz}$ for the FSU).

\begin{tabular}{|c|c|c|}
\hline & L' band $^{\prime}$ & N band \\
\hline Stellar flux (Jy) & 12.5 & 2.2 \\
Exozodiacal flux (Jy) & $0.5 \times 10^{-3}$ & $1.1 \times 10^{-3}$ \\
Initial star/EZ contrast & $2.0 \times 10^{4}$ & $2.8 \times 10^{3}$ \\
\hline Mean stellar transmission & $4.0 \times 10^{-3}$ & $5.2 \times 10^{-4}$ \\
Exozodiacal transmission & 0.37 & 0.32 \\
Final star/EZ contrast & 260 & 2.5 \\
\hline
\end{tabular}

Four possible ways to separate the exozodiacal signal from the stellar leaks have been considered:

- A priori calibration: If the stellar diameter is known with a good precision from theoretical models or previous observations, one can compute the theoretical stellar leakage and subtract this amount from the total signal in order to retrieve the signal of the exozodiacal cloud. This method has a low precision since the actual stellar transmission is generally higher than the theoretical one, because of atmospheric turbulence (as explained above). This method can thus only be used if the residual OPD is below $20 \mathrm{~nm}$ RMS. The expected calibration precision is of about $10 \%$ in that case.

- A posteriori calibration: This calibration method is an improvement of the previous one: instead of using the theoretical transmission curve, we will use the error estimates from the FSU (residual phase error) in order to compute the actual stellar transmission. This will lead to a much better calibration precision, which is still to be computed.

- Spectrum subtraction: This technique assumes that some spectral resolution will be available. The idea is to get a spectrum of the star through the interferometer in a non-nulling mode, and to subtract the stellar spectrum from the scientific data. The accuracy of such a method is highly dependent on the spectral resolution achievable (and thus on the level of the exozodiacal flux) and on the stellar type. It has not been evaluated yet.

- OPD modulation: Another way to remove the stellar contribution in the output signal is to modulate this contribution at a known frequency. This could be achieved by slightly moving the dark fringe around the centre of the field-of-view by OPD variations. The amplitude of the OPD modulation must be larger than the residual OPD from the FSU in order to get a "deterministic" modulation of the stellar leaks. The main drawback of this method is that the mean stellar leakage increases drastically, and so its associated shot noise.

In any of the four cases, further investigations are needed to assess the ultimate precision of stellar leakage calibration. Observations of exozodiacal clouds in the L' band require a very high calibration accuracy, about $0.05 \%$ in order to achieve a $10 \%$ precision on the measurement of the exozodiacal flux. The combination of spectrum subtraction and a posteriori calibration will probably be needed to reach this accuracy. On the other hand, a priori calibration would almost be sufficient in the $\mathrm{N}$ band, if the residual OPD is small enough. 


\section{CONCLUSION}

In this paper, we have shown that atmospheric turbulence is a major source of noise for a ground-based nulling interferometer, because of the fluctuations of differential optical path delay associated to atmospheric piston. The control of differential OPD is therefore an important issue, especially if near-infrared observations are considered: in the L' band $(3.5-4.1 \mu \mathrm{m})$, a residual OPD of $20 \mathrm{~nm}$ RMS is required to reduce the transmission noise below the background noise. For mid-infrared observations in the $\mathrm{N}$ band $(8-13 \mu \mathrm{m})$, a residual OPD of $400 \mathrm{~nm}$ RMS would be sufficient to achieve background-limited performances.

Another major effect of differential OPD fluctuations is to increase the mean stellar leakage. With a realistic residual OPD of $50 \mathrm{~nm}$ RMS, the amount of stellar flux in the final signal is more than twice as large as in the ideal case (with no OPD fluctuations). Four methods are proposed to calibrate the contribution stellar leakage in the output signal. Their ability to separate the exozodiacal flux from the stellar flux strongly depends on the amount of OPD fluctuations. A residual OPD of $400 \mathrm{~nm}$ RMS in the N band, which leads to a mean stellar transmission of 0.015 , would probably be too large for an accurate extraction of the scientific data. A residual OPD of $50 \mathrm{~nm}$ RMS is therefore recommended for N-band observations. This performance should be achieved by the new generation of fringe tracking units. On the other hand, nulling interferometry in the near-infrared requires the fringe tracker to be optimized for bright sources, in order to reduce the residual OPD down to $20 \mathrm{~nm}$ RMS.

\section{ACKNOWLEDGMENTS}

This Research was supported through a European Community Marie Curie Fellowship. The author is solely responsible for the information communicated, published or disseminated. It does not represent the opinion of the Community. The Community is not responsible for any use that might be made of data appearing therein.

\section{REFERENCES}

1. R. Bracewell, "Detecting nonsolar planets by spinning an infrared interferometer," Nature 274, p. 780, 1978.

2. J. Goodman, Statistical Optics, Wiley \& Sons, 1985.

3. J. Cuby, C. Lidman, and C. Moutou, "ISAAC: 18 months of Paranal Sience Operation," The Messenger 101, pp. $2-8,2000$.

4. S. Ménardi and A. Gennai, "Technical Specifications for the PRIMA Fringe Sensor Unit," tech. rep., European Southern Observatory, December 2001. VLT-SPE-ESO-15740-2210.

5. J.-M. Conan, G. Rousset, and P.-Y. Madec, "Wave-front temporal spectra in high-resolution imaging through turbulence," J. Opt. Soc. Am. 12, pp. 1559-1570, 1995. 